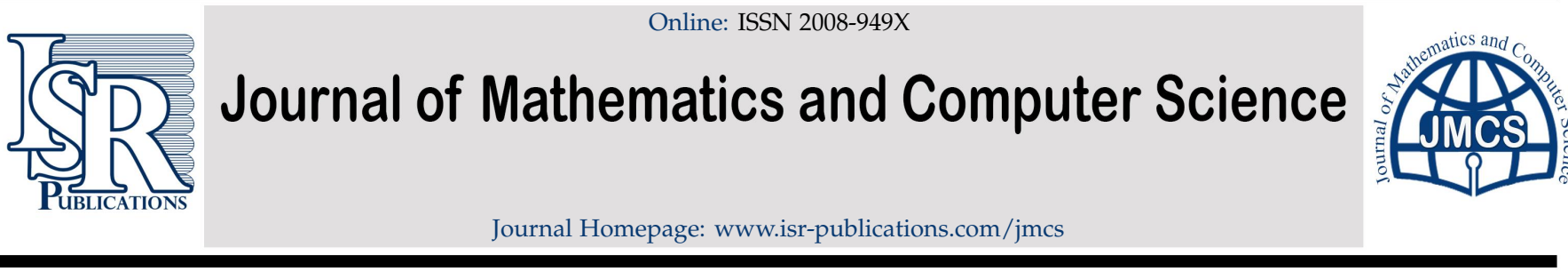

\title{
Some properties of analytical functions related to Borel dis- tribution series
}

\author{
H. Niranjan ${ }^{\mathrm{a}, *}$, A. Narasimha Murthy ${ }^{\mathrm{b}}$, P. Thirupathi Reddy \\ ${ }^{a}$ Department of Mathematics, School of Advanced Sciences, Vellore Institute of Technology, Vellore-632014, Tamilnadu, India. \\ ${ }^{b}$ Department of Mathematics, A. V. V. Junior College, Warangal-506 002, Telangana, India. \\ ${ }^{c}$ Department of Mathematics, School of Engineering, NNRESGI, Medichal-500088, Telangana, India.
}

\begin{abstract}
In this paper, we introduce and study a new subclass of analytic functions which are defined by means of a linear operator. Some results connected to coefficient estimates, growth and distortion theorems, radii of starlikeness, convexity close-toconvexity and integral means inequalities related to the subclass is obtained.
\end{abstract}

Keywords: Analytic, starlike, coefficient bounds, convexity.

2020 MSC: 30C45, 30C50.

(C2022 All rights reserved.

\section{Introduction}

Let $\mathcal{A}$ denote the class of all functions $u(z)$ of the form

$$
u(z)=z+\sum_{n=2}^{\infty} a_{n} z^{n},
$$

in the open the unit disk $E=\{z:|z|<1\}$. Let $S$ be the subclass of $\mathcal{A}$ consisting of univalent functions and satisfy the following usual normalization condition $u(0)=0$ and $u^{\prime}(0)=1$. We denote by $S$ the subclass of $\mathcal{A}$ consisting of $\mathfrak{u}(z)$ which are all univalent in $\mathrm{E}$. A function $u \in \mathcal{A}$ is a starlike function of the order $v$, $v(0 \leqslant v<1)$ if it satisfy

$$
\mathfrak{R}\left\{\frac{z u^{\prime}(z)}{u(z)}\right\}>v, \quad(z \in E),
$$

we denote by this class $\mathrm{S}^{*}(v)$.

A function $u \in \mathcal{A}$ is a convex function of the order $v, v(0 \leqslant v<1)$ if it satisfy

$$
\mathfrak{R}\left\{1+\frac{z u^{\prime \prime}(z)}{u^{\prime}(z)}\right\}>v, \quad(z \in E),
$$

we denote this class with $K(v)$.

\footnotetext{
${ }^{*}$ Corresponding author

Email addresses: hari.niranjan10@gmail.com (H. Niranjan), sparsha.adluru@gmail.com (A. Narasimha Murthy), reddypt2@gmail.com (P. Thirupathi Reddy)
}

doi: $10.22436 /$ jmcs.026.04.07

Received: 2021-11-10 Revised: 2021-11-23 Accepted: 2021-12-10 
Denote by $\mathrm{T}$ the subclass of $\mathcal{A}$ consisting of functions $u$ of the form

$$
u(z)=z-\sum_{n=2}^{\infty} a_{n} z^{n}, \quad\left(a_{n} \geqslant 0\right) .
$$

This subclass was introduced and extensively studied by Silverman[13].

For $u \in \mathcal{A}$ given by (1.1) and $g(z)$ given by

$$
g(z)=z+\sum_{n=2}^{\infty} b_{n} z^{n}
$$

their convolution (or Hadamard product), denoted by $(u * g)$, is defined as

$$
(u * g)(z)=z+\sum_{n=2}^{\infty} a_{n} b_{n} z^{n}=(g * u)(z), \quad(z \in E) .
$$

Note that $\mathrm{u} * \mathrm{~g} \in \mathcal{A}$.

\section{Mittag-Leffler function and Borel distribution}

The study of operators is fundamental in geometric function theory, complex analysis and related areas. Several derivative and integral operators can be expressed by convolution of certain analytic functions. It should be noted that this formalism helps future mathematical research as well as a better grasp of the geometric properties of such operators. Let $E_{\alpha}(z)$ and $E_{\alpha, \beta}(z)$ be functions defined by

$$
\mathrm{E}_{\alpha}(z)=\sum_{n=0}^{\infty} \frac{z^{\mathrm{n}}}{\Gamma(\alpha \mathrm{n}+1)}, \quad(z \in \mathbb{C}, \mathfrak{R}(\alpha)>0),
$$

and

$$
\mathrm{E}_{\alpha, \beta}(z)=\frac{1}{\Gamma(\beta)}+\sum_{n=1}^{\infty} \frac{z^{\mathrm{n}}}{\Gamma(\alpha \mathrm{n}+\beta)^{\prime}}, \quad(\alpha, \beta \in \mathbb{C}, \mathfrak{R}(\alpha)>0, \mathfrak{R}(\beta)>0) .
$$

It can be written in other form

$$
E_{\alpha, \beta}(z)=\frac{1}{\Gamma(\beta)}+\sum_{n=2}^{\infty} \frac{z^{n-1}}{\Gamma(\alpha(n-1)+\beta)}, \quad(\alpha, \beta \in \mathbb{C}, \mathfrak{R}(\alpha)>0, \mathfrak{R}(\beta)>0) .
$$

The function $E_{\alpha}(z)$ was introduced by Mittag-Leffler [9] and is, therefore, known as the Mittag-Leffler function. A more general function $E_{\alpha, \beta}$ generalizing $E_{\alpha}(z)$ was introduced by Wiman [18] and defined by

$$
\mathrm{E}_{\alpha, \beta}(z)=\sum_{n=0}^{\infty} \frac{z^{\mathfrak{n}}}{\Gamma(\alpha n+\beta)}, \quad(z, \alpha, \beta \in \mathrm{C}, \mathfrak{R}(\alpha)>0, \mathfrak{R}(\beta)>0) .
$$

Observe that the function $E_{\alpha, \beta}$ contains many well-known functions as its special case, for example,

$$
\begin{aligned}
& E_{1,1}(z)=e^{z}, \quad E_{1,2}(z)=\frac{e^{z}-1}{z}, \\
& E_{2,1}\left(z^{2}\right)=\cosh z \\
& E_{2,1}\left(-z^{2}\right)=\cos z \\
& E_{2,2}\left(z^{2}\right)=\frac{\sinh z}{z}
\end{aligned}
$$




$$
\begin{aligned}
& \mathrm{E}_{2,2}\left(-z^{2}\right)=\frac{\sin z}{z} \mathrm{c}, \\
& \mathrm{E}_{3}(z)=\frac{1}{2}\left[\mathrm{e}^{z^{1 / 3}}+2 e^{-\frac{1}{2} z^{1 / 3}} \cos \left(\frac{\sqrt{3}}{2} z^{1 / 3}\right)\right], \\
& \mathrm{E}_{4}(z)=\frac{1}{2}\left[\cos z^{1 / 4}+\cosh z^{1 / 4}\right] .
\end{aligned}
$$

The Mittag-Leffler function appears naturally in the solution of fractional order differential and integral equations. In the study of complex systems and super diffusive transport, in particular, fractional generalisation of the kinetic equation, random walks, and Levy flights. Several properties of Mittag-Leffler function and generalized Mittag-Leffler function can be found, e.g., in [1-3, 5-7, 12]. Observe that MittagLeffler function $\mathrm{E}_{\alpha, \beta}(z)$ does not belong to the family $\mathcal{A}$. Thus, it is natural to consider the following normalization of Mittag-Leffler functions as below

$$
\mathrm{E}_{\alpha, \beta}(z)=z \Gamma(\beta) \mathrm{E}_{\alpha, \beta}(z)=z+\sum_{n=2}^{\infty} \frac{\Gamma(\beta)}{\Gamma(\alpha(n-1)+\beta)} z^{n},
$$

it holds for complex parameters $\alpha, \beta$ and $z \in \mathbb{C}$. In this paper, we shall restrict our attention to the case of real-valued $\alpha, \beta$ and $z \in \mathrm{E}$.

A discrete random variable $x$ is said to have a Borel distribution if it takes the values $1,2,3, \ldots$ with the probabilities $\frac{e^{-\lambda}}{1 !}, \frac{2 \lambda e^{-2 \lambda}}{2 !}, \frac{9 \lambda^{2} e^{-3}}{3 !}, \cdots$, respectively, where $\lambda$ is called the parameter.

Very recently, Wanas and Khuttar [17] introduced the Borel distribution (BD) whose probability mass function is

$$
\mathrm{P}(x=\rho)=\frac{(\rho \lambda)^{\rho-1} e^{-\lambda \rho}}{\rho !}, \quad \rho=1,2,3, \cdots
$$

Wanas and Khuttar introduced a series $\mathcal{M}_{\lambda}(z)$ whose coefficients are probabilities of the Borel distribution (BD)

$$
\mathcal{M}_{\lambda}(z)=z+\sum_{n=2}^{\infty} \frac{[\lambda(n-1)]^{n-2} e^{-\lambda(n-1)}}{(n-1) !} z^{n}, \quad(0<\lambda \leqslant 1) .
$$

Murugusundaramoorthy and El-Deeb [10], El-Deeb et al. [4] and Srivastava et al. [16] are defined the Mittag-Leffler-type Borel distribution as follows:

$$
\mathcal{P}_{\lambda}(\alpha, \beta ; \rho)=\frac{(\lambda \rho)^{\rho-1}}{E_{\alpha, \beta}(\lambda \rho) \Gamma(\alpha \rho+\beta)}, \quad \rho=0,1,2, \cdots,
$$

where

$$
E_{\alpha, \beta}(z)=\sum_{n=0}^{\infty} \frac{z^{n}}{\Gamma(\alpha n+\beta)}, \quad(\alpha, \beta \in \mathbb{C}, \mathfrak{R}(\alpha)>0, \mathfrak{R}(\beta)>0) .
$$

Thus by using (1.7) and (1.8) and by convolution operator, the Mittag-Leffler-type Borel distribution series defined as below

$$
\mathrm{B}_{\lambda}(\alpha, \beta)(z)=z+\sum_{n=2}^{\infty} \frac{[\lambda(n-1)] ![\lambda(n-1)]^{n-2} e^{-\lambda(n-1)}}{(n-1) ! E_{\alpha, \beta}(\lambda(n-1)) \Gamma(\alpha(n-1)+\beta)} z^{n}, \quad(0<\lambda \leqslant 1) .
$$

Furthermore, we consider the linear operator $\mathrm{B}_{\lambda}(\alpha, \beta): \mathcal{A} \rightarrow \mathcal{A}$ defined by the convolution or Hadamard product 


$$
\begin{aligned}
\mathrm{B}_{\lambda}(\alpha, \beta) u(z) & =\mathrm{B}_{\lambda}(\alpha, \beta)(z) * u(z) \\
& =z+\sum_{n-2}^{\infty} \frac{[\lambda(n-1)] ![\lambda(n-1)]^{n-2} e^{-\lambda(n-1)}}{(n-1) ! \mathrm{E}_{\alpha, \beta}(\lambda(n-1)) \Gamma(\alpha(n-1)+\beta)} a_{n} z^{n} \\
& =z+\sum_{n=2}^{\infty} \phi(n) a_{n} z^{n}, \quad(\alpha, \beta \in \mathbb{C}, \mathfrak{R}(\alpha)>0, \mathfrak{R}(\beta)>0,0<\lambda \leqslant 1),
\end{aligned}
$$

where

$$
\phi(n)=\frac{[\lambda(n-1)] ![\lambda(n-1)]^{n-2} e^{-\lambda(n-1)}}{(n-1) ! E_{\alpha, \beta}(\lambda(n-1)) \Gamma(\alpha(n-1)+\beta)} .
$$

If $u \in T$ is given by (1.1) then we have

$$
\mathrm{B}_{\lambda}(\alpha, \beta) u(z)=z-\sum_{n=2}^{\infty} \phi(n) a_{n} z^{n},
$$

where $\phi(n)$ is given by (1.10). Now, by making use of the Mittag-Leffler-type Borel distribution series $\mathrm{B}_{\lambda}(\alpha, \beta)$, we define a new subclass of functions belonging to the class $\mathcal{A}$.

Now, we define the following new subclass motivated by Popade et al [11].

Definition 1.1. The function $u(z)$ of the form (1.1) is in the class $S_{\lambda}(\alpha, \beta, \mu, \gamma)$, if it satisfies the inequality

$$
\mathfrak{R}\left\{\frac{z\left(B_{\lambda}(\alpha, \beta) u(z)\right)^{\prime}}{(1-\mu) z+\mu B_{\lambda}(\alpha, \beta) u(z)}-\alpha\right\}>\left|\frac{z\left(B_{\lambda}(\alpha, \beta) u(z)\right)^{\prime}}{(1-\mu) z+\mu B_{\lambda}(\alpha, \beta) u(z)}-1\right|,
$$

for $0 \leqslant \mu \leqslant 1,0 \leqslant \gamma \leqslant 1$. Further we define $\operatorname{TS}_{\lambda}(\alpha, \beta, \mu, \gamma)=S_{\lambda}(\alpha, \beta, \mu, \gamma) \cap \mathrm{T}$.

The aim of present paper is to study the coefficient bounds, radii of close-to-convex and starlikeness convex linear combinations and integral means inequalities of the $\operatorname{TS}_{\lambda}(\alpha, \beta, \mu, \gamma)$.

\section{Coefficient bounds}

Theorem 2.1. A function $u(z)$ of the form (1.1) is in $S_{\lambda}(\alpha, \beta, \mu, \gamma)$, then

$$
\sum_{n=2}^{\infty}[2 n-\mu(\gamma+1)] \phi(n)\left|a_{n}\right| \leqslant 1-\gamma,
$$

where $0 \leqslant \mu \leqslant 1,0 \leqslant \gamma \leqslant 1$ and $\phi(n)$ is given by (1.10).

Proof. It suffices to show that

$$
\left|\frac{z\left(B_{\lambda}(\alpha, \beta) u(z)\right)^{\prime}}{(1-\mu) z+\mu B_{\lambda}(\alpha, \beta) u(z)}-1\right|-\Re\left\{\frac{z\left(B_{\lambda}(\alpha, \beta) u(z)\right)^{\prime}}{(1-\mu) z+\mu B_{\lambda}(\alpha, \beta) u(z)}-1\right\} \leqslant 1-\gamma .
$$

We have

$$
\begin{aligned}
\left|\frac{z\left(B_{\lambda}(\alpha, \beta) u(z)\right)^{\prime}}{(1-\mu) z+\mu B_{\lambda}(\alpha, \beta) u(z)}-1\right|- & \Re\left\{\frac{z\left(B_{\lambda}(\alpha, \beta) u(z)\right)^{\prime}}{(1-\mu) z+\mu B_{\lambda}(\alpha, \beta) u(z)}-1\right\} \\
& \leqslant 2\left|\frac{z\left(B_{\lambda}(\alpha, \beta) u(z)\right)^{\prime}}{(1-\mu) z+\mu B_{\lambda}(\alpha, \beta) u(z)}-1\right| \\
& \leqslant \frac{2 \sum_{n=2}^{\infty}(n-\mu) \phi(n)\left|a_{n}\right||z|^{n-1}}{1-\sum_{n=2}^{\infty} \mu \phi(n)\left|a_{n}\right||z|^{n-1}} \\
& \leqslant \frac{2 \sum_{n=2}^{\infty}(n-\mu) \phi(n)\left|a_{n}\right|}{1-\sum_{n=2}^{\infty} \mu \phi(n)\left|a_{n}\right|}
\end{aligned}
$$


The last expression is bounded above by $(1-\gamma)$ if

$$
\sum_{n=2}^{\infty}[2 n-\mu(\gamma+1)] \phi(n)\left|a_{n}\right| \leqslant 1-\gamma,
$$

and the proof is complete.

Theorem 2.2. Let $0 \leqslant \mu \leqslant 1,0 \leqslant \gamma \leqslant 1$, then a function $u$ of the form (1.4) to be in the class $\operatorname{TS}_{\lambda}(\alpha, \beta, \mu, \gamma)$ if and only if

$$
\sum_{n=2}^{\infty}[2 n-\mu(\gamma+1)] \phi(n)\left|a_{n}\right| \leqslant 1-\gamma
$$

where $\phi(\mathrm{n})$ is given by (1.10).

Proof. In view of Theorem 2.1 we need only to prove the necessity. If $u \in \operatorname{TS}_{\lambda}(\alpha, \beta, \mu, \gamma)$ and $z$ is real, then

$$
\Re\left\{\frac{1-\sum_{n=2}^{\infty} n \phi(n) a_{n} z^{n-1}}{1-\sum_{n=2}^{\infty} \mu \phi(n) a_{n} z^{n-1}}-\gamma\right\}>\left|\frac{\sum_{n=2}^{\infty}(n-\mu) \phi(n) a_{n} z^{n-1}}{1-\sum_{n=2}^{\infty} \mu \phi(n) a_{n} z^{n-1}}\right| .
$$

Letting $z \rightarrow 1$ along the real axis, we obtain the desired inequality

$$
\sum_{n=2}^{\infty}[2 n-\mu(\gamma+1)] \phi(n)\left|a_{n}\right| \leqslant 1-\gamma,
$$

where $0 \leqslant \mu \leqslant 1,0 \leqslant \gamma \leqslant 1$ and $\phi(n)$ is given by (1.10).

Corollary 2.3. If $u(z) \in \operatorname{TS}_{\lambda}(\alpha, \beta, \mu, \gamma)$, then

$$
\left|a_{n}\right| \leqslant \frac{1-\gamma}{[2 n-\mu(\gamma+1)] \phi(n)}
$$

where $0 \leqslant \mu \leqslant 1,0 \leqslant \gamma \leqslant 1$ and $\phi(n)$ is given by (1.10). Equality holds for the function

$$
u(z)=z-\frac{1-\gamma}{[2 n-\mu(\gamma+1)] \phi(n)} z^{n}
$$

Theorem 2.4. Let $u_{1}(z)=z$ and

$$
u_{n}(z)=z-\frac{1-\gamma}{[2 n-\mu(\gamma+1)] \phi(n)} z^{n}, \quad n \geqslant 2 .
$$

Then $\mathrm{u}(z) \in \mathrm{TS}_{\lambda}(\alpha, \beta, \mu, \gamma)$, if and only if, it can be expressed in the form

$$
u(z)=\sum_{n=1}^{\infty} w_{n} u_{n}(z), \quad w_{n} \geqslant 0, \quad \sum_{n=1}^{\infty} w_{n}=1 .
$$

Proof. Suppose $u(z)$ can be written as in (2.4), then

$$
u(z)=z-\sum_{n=2}^{\infty} w_{n} \frac{1-\gamma}{[2 n-\mu(\gamma+1)] \phi(n)} z^{n}
$$


Now,

$$
\sum_{n=2}^{\infty} w_{n} \frac{(1-\gamma)[2 n-\mu(\gamma+1)] \phi(n)}{(1-\gamma)[2 n-\mu(\gamma+1)] \phi(n)}=\sum_{n=2}^{\infty} w_{n}=1-w_{1} \leqslant 1 .
$$

Thus $u(z) \in \operatorname{TS}_{\lambda}(\alpha, \beta, \mu, \gamma)$.

Conversely, let $u(z) \in \operatorname{TS}_{\lambda}(\alpha, \beta, \mu, \gamma)$, then by using (2.2), we get

$$
w_{n}=\frac{[2 n-\mu(\gamma+1)] \phi(n)}{(1-\gamma)} a_{n}, \quad n \geqslant 2,
$$

and $w_{1}=1-\sum_{n=2}^{\infty} w_{n}$. Then we have $u(z)=\sum_{n=1}^{\infty} w_{n} u_{n}(z)$ and hence this completes the proof of Theorem.

Theorem 2.5. The class $\operatorname{TS}_{\lambda}(\alpha, \beta, \mu, \gamma)$ is a convex set.

Proof. Let the function

$$
u_{j}(z)=z-\sum_{n=2}^{\infty} a_{n, j} z^{n}, \quad a_{n, j} \geqslant 0, \quad j=1,2,
$$

be in the class $\operatorname{TS}_{\lambda}(\alpha, \beta, \mu, \gamma)$. It is sufficient to show that the function $h(z)$ defined by

$$
h(z)=\xi \mathfrak{u}_{1}(z)+(1-\xi) u_{2}(z), 0 \leqslant \xi<1,
$$

in the class $\operatorname{TS}_{\lambda}(\alpha, \beta, \mu, \gamma)$. Since

$$
h(z)=z-\sum_{n=2}^{\infty}\left[\xi a_{n, 1}+(1-\xi) a_{n, 2}\right] z^{n} .
$$

An easy computation with the aid of Theorem (2.2) gives

$$
\begin{aligned}
\sum_{n=2}^{\infty}[2 n-\mu(\gamma+1)] \xi \phi(n) a_{n, 1}+ & \sum_{n=2}^{\infty}[2 n-\mu(\gamma+1)](1-\xi) \phi(n) a_{n, 2} \\
& \leqslant \xi(1-\gamma)+(1-\xi)(1-\gamma)=(1-\gamma),
\end{aligned}
$$

which implies that $h \in \operatorname{TS}_{\lambda}(\alpha, \beta, \mu, \gamma)$. Hence $\operatorname{TS}_{\lambda}(\alpha, \beta, \mu, \gamma)$ is convex.

\section{Radii of close-to-convexity, starlikeness and convexity}

In this section, we obtain the radii of close-to-convexity, starlikeness and convexity for the class $\operatorname{TS}_{\lambda}(\alpha, \beta, \mu, \gamma)$.

Theorem 3.1. Let the function $\mathrm{u}(z)$ defined by (1.4) belong to the class $\operatorname{TS}_{\lambda}(\alpha, \beta, \mu, \gamma)$, then $\mathrm{u}(z)$ is close-to-convex of order $\delta(0 \leqslant \delta<1)$ in the disc $|z|<r_{1}$, where

$$
r_{1}=\inf _{n \geqslant 2}\left[\frac{(1-\delta) \sum_{n=2}^{\infty}[2 n-\mu(\gamma+1)] \phi(n)}{n(1-\gamma)}\right]^{1 / n-1}, n \geqslant 2 .
$$

The result is sharp, with the external function $\mathrm{u}(z)$ is given by (2.3). 
Proof. Given $u \in T$ and $u$ is close-to-convex of order $\delta$, we have

$$
\left|\mathfrak{u}^{\prime}(z)-1\right|<1-\delta
$$

For the left hand side of (3.1), we have

$$
\left|u^{\prime}(z)-1\right| \leqslant \sum_{n=2}^{\infty} n a_{n}|z|^{n-1} .
$$

The last expression is less than $1-\delta$

$$
\sum_{n=2}^{\infty} \frac{n}{1-\delta} a_{n}|z|^{n-1} \leqslant 1 .
$$

Using the fact, that $u(z) \in \operatorname{TS}_{\lambda}(\alpha, \beta, \mu, \gamma)$ if and only if

$$
\sum_{n=2}^{\infty} \frac{[2 n-\mu(\gamma+1)] \phi(n)}{1-\gamma} a_{n} \leqslant 1 .
$$

We can see that (3.1) is true, if

$$
\frac{n}{1-\delta}|z|^{n-1} \leqslant \frac{[2 n-\mu(\gamma+1)] \phi(n)}{1-\gamma}
$$

or, equivalently

$$
|z| \leqslant\left\{\frac{(1-\delta)[2 n-\mu(\gamma+1)] \phi(n)}{n(1-\gamma)}\right\}^{1 / n-1}
$$

which completes the proof.

Theorem 3.2. Let the function $u(z)$ defined by (1.4) belong to the class $\operatorname{TS}_{\lambda}(\alpha, \beta, \mu, \gamma)$. Then $u(z)$ is starlike of order $\delta(0 \leqslant \delta<1)$ in the disc $|z|<r_{2}$, where

$$
r_{2}=\inf _{n \geqslant 2}\left[\frac{(1-\delta) \sum_{n=2}^{\infty}[2 n-\mu(\gamma+1)] \phi(n)}{(n-\delta)(1-\gamma)}\right]^{1 / n-1} .
$$

The result is sharp, with external function $\mathrm{u}(z)$ is given by (2.3).

Proof. Given $u \in T$ and $u$ is starlike of order $\delta$, we have

$$
\left|\frac{z u^{\prime}(z)}{u(z)}-1\right|<1-\delta
$$

For the left hand side of (3.2), we have

$$
\left|\frac{z u^{\prime}(z)}{u(z)}-1\right| \leqslant \sum_{n=2}^{\infty} \frac{(n-1) a_{n}|z|^{n-1}}{1-\sum_{n=2}^{\infty} a_{n}|z|^{n-1}} .
$$

The last expression is less than $1-\delta$ if

$$
\sum_{n=2}^{\infty} \frac{n-\delta}{1-\delta} a_{n}|z|^{n-1}<1
$$


Using the fact that $u(z) \in \operatorname{TS}_{\lambda}(\alpha, \beta, \mu, \gamma)$ if and only if

$$
\sum_{n=2}^{\infty} \frac{[2 n-\mu(\gamma+1)] \phi(n)}{1-\gamma} a_{n} \leqslant 1
$$

We can say (3.2) is true, if

$$
\sum_{n=2}^{\infty} \frac{n-\delta}{1-\delta}|z|^{n-1} \leqslant \frac{[2 n-\mu(\gamma+1)] \phi(n)}{1-\gamma}
$$

or equivalently

$$
|z|^{n-1} \leqslant \frac{(1-\delta)[2 n-\mu(\gamma+1)] \phi(n)}{(n-\delta)(1-\gamma)}
$$

which yields the starlikeness of the family.

\section{Integral means inequalities}

In [13], Silverman found that the function $u_{2}(z)=z-\frac{z^{2}}{2}$ is often extremal over the family T. He applied this function to resolve his integral means inequality conjunctured [14] and setteled in [15], that

$$
\int_{0}^{2 \pi}\left|u\left(r e^{i \varphi}\right)\right|^{\tau} d \varphi \leqslant \int_{0}^{2 \pi}\left|u_{2}\left(r e^{i \varphi}\right)\right|^{\tau} d \varphi,
$$

for all $u \in T, \tau>0$ and $0<r<1$. In [15], he also proved his conjuncture for the subclasses $T^{*}(\alpha)$ and $C(\alpha)$ of $T$.

Now, we prove Silverman's conjecture for the class of functions $\operatorname{TS}_{\lambda}(\alpha, \beta, \mu, \gamma)$. We need the concept of subordination between analytic functions and a subordination theorem of Littlewood [8].

Two functions $u$ and $v$, which are analytic in $E$, the function $u$ is said to be subordinate to $v$ in $E$, if there exists a function $w$ analytic in $\mathrm{E}$ with $w(0)=0,|w(z)|<1,(z \in \mathrm{E})$ such that $u(z)=v(w(z)),(z \in \mathrm{E})$. We denote this subordination by $u(z) \prec v(z)$. ( $\prec$ denote subordination)

Lemma 4.1. If the function $\mathrm{u}$ and $v$ are analytic in $\mathrm{E}$ with $\mathrm{u}(z) \prec v(z)$, then for $\tau>0$ and $z=r e^{i \varphi}, 0<\mathrm{r}<1$

$$
\int_{0}^{2 \pi}\left|v\left(r e^{i \varphi}\right)\right|^{\tau} \mathrm{d} \varphi \leqslant \int_{0}^{2 \pi}\left|u\left(r e^{i \varphi}\right)\right|^{\tau} \mathrm{d} \varphi .
$$

Now, we discuss the integral means inequalities for functions $u$ in $\operatorname{TS}_{\lambda}(\alpha, \beta, \mu, \gamma)$.

Theorem 4.2. $u \in \operatorname{TS}_{\lambda}(\alpha, \beta, \mu, \gamma), 0 \leqslant \mu<1,0 \leqslant \gamma<1$ and $u_{2}(z)$ be defined by

$$
\mathrm{u}_{2}(z)=z-\frac{1-\gamma}{\phi(2)} z^{2}
$$

Proof. For $u(z)=z-\sum_{n=2}^{\infty} a_{n} z^{n},(4.1)$ is equivalent to

$$
\int_{0}^{2 \pi}\left|1-\sum_{n=2}^{\infty} a_{n} z^{n-1}\right|^{\tau} d \varphi \leqslant \int_{0}^{2 \pi}\left|1-\frac{1-\gamma}{\varphi(2)} z\right|^{\tau} \mathrm{d} \varphi .
$$

By Lemma (4.1), it is enough to prove that

$$
1-\sum_{n=2}^{\infty} a_{n} z^{n-1} \prec 1-\frac{1-\gamma}{\varphi(2)} z .
$$


Assuming

$$
1-\sum_{n=2}^{\infty} a_{n} z^{n-1} \prec 1-\frac{1-\gamma}{\varphi_{2}} w(z),
$$

and using (2.1), we obtain

$$
|w(z)|=\left|\sum_{n=2}^{\infty} \frac{\varphi(2)}{1-\gamma} a_{n} z^{n-1}\right| \leqslant|z| \sum_{n=2}^{\infty} \frac{\varphi(2)}{1-\gamma} a_{n} \leqslant|z|,
$$

where

$$
\varphi(n)=[2 n-\mu(\gamma+1) \phi(n) .
$$

This completes the proof.

\section{Concluding remarks}

This research has introduced study the Mittag-Leffler-type Borel distribution series related to analytic function and studied some basic properties of geometric function theory. Accordingly, some results to coefficient estimates, growth and distortion theorem, radii of starlikeness, convexity, close-to-convexity and integral means inequalities have also been considered, inviting future research for this field of study. We hope that this distribution series play a significant role in several branches of Mathematics, Science and Technology.

\section{Acknowledgment}

The authors express their sincere thanks to the esteemed referee(s) for their careful readings, valuable suggestions and comments, which helped them to improve the presentation of the paper.

\section{References}

[1] N. Alessa, B. Venkateswarlu, P. Thirupathi Reddy, K. Loganathan, K. Tamilvanan, A New subclass of analytic functions related to Mittag-Leffler type Poisson distribution series, J. Funct. Spaces, 2021 (2021), 7 pages. 1

[2] A. A. Attiya, Some applications of Mittag-Leffler function in the unit disk, Filomat, 30 (2016), 2075-2081.

[3] D. Bansal, J. K. Prajapat, Certain geometric properties of the Mittag-Leffler functions, Complex Var. Elliptic Equ., 61 (2016), 338-350. 1

[4] S. M. El-Deeb, G. Murugusundaramoorthy, A. Alburaikan, Bi-Bazilevic functions based on the Mittag-Leffler-type Borel distribution associated with Legendre polynomials, J. Math. Computer Sci., 24 (2022), 235-245. 1

[5] B. A. Frasin, An application of an operator associated with generalized Mittag-Leffler function, Konuralp J. Math., 7 (2019), 199-202. 1

[6] B. A. Frasin, T. Al-Hawary, F. Yousef, Some properties of a linear operator involving generalized Mittag-Leffler function, Stud. Univ. Babes-Bolyai Math., 65 (2020), 67-75.

[7] M. Grag, P. Manohar, S. L. Kalla, A Mittag-Leffler-type function of two variables, Integral Transforms Spec. Funct., 24 (2013), 934-944. 1

[8] J. E. Littlewood, On inequalities in the theory of functions, Proc. London Math. Soc. (2), 23 (1925), 481-519. 4

[9] G. Mittag-Leffler, Sur la Nouvelle Fonction $\mathrm{E}_{\alpha}(\mathrm{x})$, Comptes Rendus de l'Academie des Sciences Paris, 137 (1903), 554-558. 1

[10] G. Murugusundaramoorthy, S. M. El-Deeb, Second Hankel determinant for a class of analytic functions of the MittagLeffler-type Borel distribution related with Legendre polynomials, Turkish World Math. Soc. J. Appl. Eng. Math., accepted for publications, (2021). 1

[11] S. M. Popade, R. N. Ingle, P. T. Reddy, On a certain subclass of analytic functions defined by a differential operator, Malaya J. Mat., 8 (2020), 576-580. 1

[12] K. A. Selvakumaran, H. A. Al-Kharsani, D. Baleanu, S. D. Purohi, K. S. Nisar, Inclusion relationships for some subclasses of analytic functions associated with generalized Bessel functions, J. Comput. Anal. Appl., 24 (2018), 81-90. 1

[13] H. Silverman, Univalent functions with negative coefficients, Proc. Amer. Math. Soc., 51 (1975), 109-116. 1, 4

[14] H. Silverman, A survey with open problems on univalent functions whose coefficient are negative, Rocky Mountain J. Math., 21 (1991), 1099-1125. 4 
[15] H. Silvermani, Integral means for univalent functions with negative coefficient, Houston J. Math., 23 (1997), 169-174. 4

[16] H. M. Srivastava, G. Murugusundaramoorthy, S. M. El-Deeb, Faber polynomial coefficient estimates of bi-close-toconvex functions connected with the Borel distribution of the Mittag-leffler type, J. Nonlinear Var. Anal., 5 (2021), 103118. 1

[17] A. K. Wanas, J. A. Khuttar, Applications of Borel distribution series on analytic functions, Earthline J. Math. Sci., 4 (2020), 71-82. 1

[18] A. Wiman, Über die Nullstellen der Funktionen $\mathrm{E}_{\mathrm{a}}(\mathrm{x})$, Acta Math., 29 (1905), 217-134. 1 